\title{
Haemoperitoneum Due to Spontaneous Rupture of a Liver Metastasis
}

\author{
Christodoulos Dolapsakis ${ }^{1}$, Polina Pavli ${ }^{1}$, Andreas Panagopoulos ${ }^{1}$, Ekaterini Tavernaraki ${ }^{2}$, Georgios Varnavas ${ }^{1}$, \\ Androniki Papapostolou ${ }^{1}$, Alexandros Machairas ${ }^{1}$ \\ ${ }^{1} 3$ rd Department of Internal Medicine, Evangelismos General Hospital, Athens, Greece \\ ${ }^{2}$ Radiology Department, Evangelismos General Hospital, Athens, Greece
}

Received: 02/05/2019

Accepted: 16/05/2019

Published: 26/06/2019

\begin{abstract}
How to cite this article: Dolapsakis C, Pavli P, Panagopoulos A, Tavernaraki E, Varnavas G, Papapostolou A, Machairas A. Haemoperitoneum due to spontaneous rupture of a liver mestastasis. EJCRIM 2019;6: doi:10.12890/2019_001142.
\end{abstract}

Conflicts of Interests: The Authors declare that there are no competing interest

This article is licensed under a Commons Attribution Non-Commercial 4.0 License

\section{ABSTRACT}

Spontaneous rupture of a liver metastasis is a life-threatening complication of metastatic liver disease. Although metastatic liver lesions are much more common than primary tumours, spontaneous rupture of a liver metastasis is rare. Therapeutic decisions must take into account the extent of metastatic liver disease and the patient's performance status. Transarterial embolization may be considered in cases of ongoing haemorrhage despite initial conservative measures. We describe a case of haemoperitoneum due to spontaneous rupture of a liver metastasis in a 72-year-old patient with carcinoma of unknown origin who responded well to conservative management.

\section{LEARNING POINTS}

- Spontaneous rupture of a liver metastasis is a rare but potentially life-threatening complication of metastatic cancer to the liver; risk factors include subcapsular location, rapid tumour growth and tumour necrosis (spontaneous or due to chemotherapy).

- Unexplained fever often precedes the spontaneous rupture, probably reflecting tumour necrosis or infiltration of the liver capsule, and may raise clinical suspicion for the diagnosis.

- Transarterial embolization may be considered in cases of ongoing haemorrhage despite initial conservative measures.

\section{KEYWORDS}

Liver, metastasis, rupture, haemoperitoneum

\section{CASE DESCRIPTION}

A 72-year-old woman was admitted to our hospital because of fever. Six months before admission, she had been diagnosed with metastatic carcinoma of unknown origin after a biopsy of an osteolytic lesion of the left femur. At that time, computed tomography (CT) of the chest and abdomen and a bone scan revealed extensive metastatic disease in the lung parenchyma, liver and bones (thoracic and lumbar spine, ribs, sternum, skull and femur). The patient underwent chemotherapy and palliative radiotherapy. One month before admission, a followup CT scan showed progression of the metastatic lesions of the bones and liver. Palliative radiotherapy was started and chemotherapy was deferred as the patient had a very poor performance status as she was confined to bed due to severe bone pain. She was admitted to our hospital a week after completion of the radiotherapy.

At admission, the patient had fever but was otherwise stable, without symptoms or signs of infection. Laboratory studies showed a white cell count (WBC) of $11.5 \times 10^{9} / \mathrm{l}$, a haemoglobin level of $9.7 \mathrm{~g} / \mathrm{dl}$ and C-reactive protein (CRP) of $14.9 \mathrm{mg} / \mathrm{dl}$ (reference range $<0.5 \mathrm{mg} / \mathrm{dl}$ ). Chest radiography did not show any pulmonary infiltrates and blood and urine cultures were sterile. On the $6^{\text {th }}$ day of admission, the patient suddenly developed hypotension and tachycardia, and haemoglobin dropped to $6 \mathrm{~g} / \mathrm{dl}$ without evidence of blood loss. At clinical examination, there was tenderness during palpation of the right upper quadrant. 
Coagulation tests and fibrinogen were within normal limits. Abdominal ultrasound detected free intraperitoneal fluid. This was followed by abdominal CT angiography (Fig. 1) which confirmed the presence of haemoperitoneum and revealed a subcapsular hepatic haematoma adjacent to a metastatic lesion in liver segments II and III, without extravasation of contrast. On the basis of these findings, a diagnosis of spontaneous rupture of a hepatic metastasis with haemoperitoneum was made.

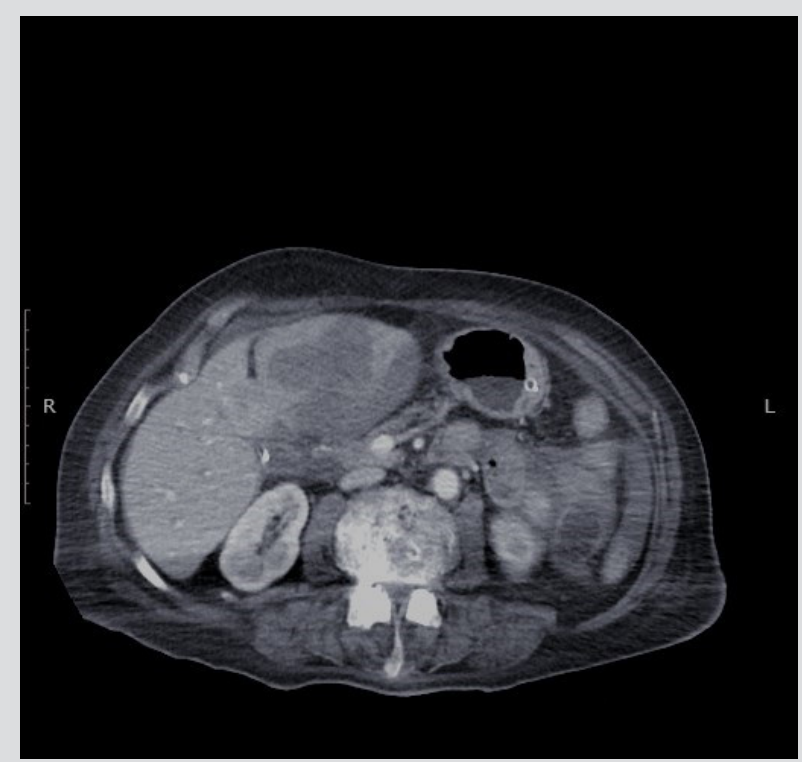

Figure 1. Axial CT scan of the abdomen showing a subcapsular hepatic haematoma adjacent to a metastatic lesion situated in liver segments II and III, without extravasation of contrast

Taking into account the absence of active bleeding on CT and the poor overall status and prognosis of the patient, conservative management was instituted. Crystalloid fluids and 4 units of packed red blood cells were administered. The patient's haemodynamic status improved, haemoglobin remained stable and the fever subsided. The patient was discharged home on the $15^{\text {th }}$ day of admission.

\section{DISCUSSION}

Spontaneous rupture of a liver metastasis is very rare compared with hepatocellular carcinoma (HCC) rupture, even though metastatic hepatic lesions are much more common than HCC. This probably reflects the fact that hepatic metastases have less vascularity than HCC lesions and tend to be more fibrotic and less penetrative to the liver capsule. Various primary tumours have been reported in cases of liver metastases rupture including those of the lung, pancreas, skin, prostate, kidney, testis and stomach. Possible risk factors for metastasis rupture include subcapsular location, rapid tumour growth and tumour necrosis (either spontaneous or resulting from chemotherapy) ${ }^{[1,2]}$.

The signs and symptoms of spontaneous rupture of a liver metastasis depend on the extent of bleeding and vary from subtle abdominal pain to haemorrhagic shock and acute abdomen in cases of haemoperitoneum. Unexplained fever often precedes the abrupt appearance of haemoperitoneum ${ }^{[2]}$, which may reflect tumour necrosis or infiltration of the liver capsule and may raise clinical suspicion for the diagnosis in the appropriate clinical setting. Emergency abdominal ultrasound is usually the first examination performed, and shows free intraperitoneal fluid. However, it may be unrevealing in cases of pre-existing ascites. There are reports that contrast-enhanced ultrasonography can confirm active bleeding and determine the location of the bleeding lesion if contrast is present in the ascites ${ }^{[2]}$. CT angiography is the diagnostic modality of choice and may shows signs of ongoing bleeding with extravasation of contrast. It also offers useful information regarding the overall prognosis, such as signs of cirrhosis, portal hypertension, portal vein thrombosis and the extent of the metastatic disease in the liver. The short-term prognosis of spontaneous rupture of a liver metastasis is determined by bleeding severity, while long-term prognosis depends on the cancer stage and the patient's performance status. Therapeutic options are limited in cases of advanced cancer, multiple liver metastatic lesions and poor performance status. In the absence of active bleeding on CT angiography, conservative management is appropriate and will achieve haemostasis in many cases. The role of transarterial embolization (TAE) is well established in HCC haemorrhage ${ }^{[3]}$ and there are case reports of its successful use in ruptured liver metastases ${ }^{[1,4]}$. Patients with ongoing bleeding and oligometastatic liver disease with a good performance status are the most promising candidates for this approach. Surgical resection of liver metastasis carries significant mortality in the emergency setting but may be considered after haemostasis has been achieved, especially in cases of solitary liver metastasis ${ }^{[3,5]}$. 


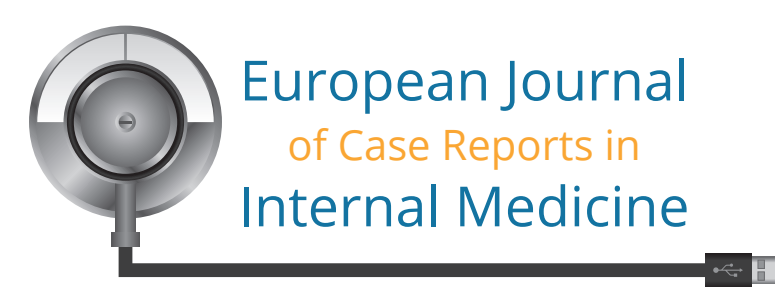

In conclusion, spontaneous rupture of a liver metastasis, although rare, must be considered in patients with metastatic liver disease with abdominal pain, anaemia or haemorrhagic shock, especially if otherwise unexplained fever had preceded the abrupt appearance of these symptoms.

\section{REFERENCES}

1. Mochimaru T, Minematsu N, Ohsawa K, Tomomatsu K, Miura H, Betsuyaku T, et al. Hemoperitoneum secondary to rupture of a hepatic metastasis from small cell lung cancer during chemotherapy: a case with a literature review. Intern Med 2017;56:695-699.

2. Naganuma H, Funaoka M, Fujimori S, Niwa M, Hirano H, Ishida H, et al. Rupture of liver metastasis: report of a case with an emphasis on contrast-enhanced US. J Med Ultrason (2001) 2007;34:113-116.

3. Srinivasa S, Lee WG, Aldameh A, Koea JB. Spontaneous hepatic haemorrhage: a review of pathogenesis, aetiology and treatment. HPB (Oxford) 2015;17:872-880.

4. Sakai M, Oguri T, Sato S, Hattori N, Bessho Y, Achiwa H, et al. Spontaneous hepatic rupture due to metastatic tumor of lung adenocarcinoma. Intern Med 2005;44:50-54.

5. Kim HJ, Park YE, Ki MS, Lee SJ, Beom SH, Han DH, et al. Spontaneous rupture of hepatic metastasis from a thymoma: a case report. World J Gastroenterol 2016;22:9860-9864. 